\title{
EL ENTRENAMIENTO COMO COMPONENTE PARA UN PROCESO DE TRANSFORMACIÓN INSTITUCIONAL
}

\author{
Grab. Fabián Fuel Revelo ${ }^{1}$
}

\begin{abstract}
Resumen
El presente trabajo se encuadra en el proceso de transformación que viene desarrollando el Ejército Ecuatoriano y describe la investigación realizada para establecer una base para la Línea de Transformación (LDT) "entrenamiento”, tomando en cuenta el modelo de entrenamiento actual comparado con el de otros países; la cultura física, con sus respectivos promedios obtenidos tanto de oficiales como de tropa, y los centros de entrenamiento disponibles en el ejército. Una vez establecida esta línea base en función de la información obtenida en el Comando de Operaciones Terrestre se conceptualiza la LDT "entrenamiento" como: "Mejorar los actuales modelos de entrenamiento, cultura física y entrenamiento físico militar individual y colectivo, a través de modernas metodologías y la implementación de centros de entrenamiento que permitan incrementar el alistamiento operacional de las unidades militares para hacer frente a los escenarios actuales y futuros". Establecido el diagnóstico, se proponen objetivos y estrategias para las diferentes etapas que conforman el plan de transformación, el cual determina cuatro períodos iniciando en el año 2019 y concluyendo en el 2033; finalmente, se determina un gráfico de convergencia entre el relacionamiento de la LDT "entrenamiento" con otras LDT establecidas en el Plan de Transformación de Ejército. La presente investigación descubre aspectos muy importantes que, plasmados en los objetivos y estrategias, fortalecerá al Plan de Transformación de Ejército, lo cual permitirá diseñar programas y proyectos en beneficio del alistamiento operacional y con ello el cumplimiento de la misión del Ejército. La LDT "entrenamiento" definitivamente es uno de los pilares fundamentales de la transformación. Solamente la voluntad, compromiso y continuidad del proyecto permitirá la transformación del Ejército para estar en mejores condiciones de enfrentar los nuevos desafíos a la seguridad y defensa.
\end{abstract}

Palabras clave: Ejército, Transformación, Entrenamiento.

\begin{abstract}
This work is part of the transformation process that the Ecuadorian Army has been developing and describes the research carried out to establish a baseline for the Transformation Line (LDT) "training", taking into account the current training model compared to that of other countries; physical culture, with their respective averages obtained from both officers and troops; and, the training centers available in the Army. Once this baseline has been established based on the information obtained in the Land Operations Command, the LDT "training" is conceptualized as: "Improving the current training models, physical culture and individual and collective military physical training, through modern methodologies and the implementation of training centers, which allow increasing the operational readiness of military units to face current and future scenarios". Once the diagnosis has been established, objectives and strategies are proposed for the different periods of time that appear in the transformation plan, which determines four periods, whose beginning is 2019 and ending in 2033; to finally determine in a convergence graph, between the relationship of the LDT "training" with other LDT established in the Army Transformation Plan. The contribution of this research reveals very important aspects that, reflected in the objectives and strategies, will strengthen the Army Transformation Plan, which will allow the design of programs and projects for the benefit of operational enlistment and with it the fulfillment of the Army's mission. The LDT "training" is definitely one of the fundamental pillars of transformation. Only the will, commitment and continuity of the project will allow the transformation of the Army and thus be in better conditions to face the new challenges to security and defense.
\end{abstract}

Keywords : Army, Transformation, Training.

\footnotetext{
${ }^{1}$ fuelf18@yahoo.com

EJÉRCITO ECUATORIANO
}

DOI: https://dx.doi.org/10.24133/age.n14.2021.10

Revista Academia de Guerra del Ejército Ecuatoriano, Volumen 14. Núm. 1 abril 2021. pp. 112 - 124 


\section{Introducción}

$\mathrm{E}_{\mathrm{p}}^{1}$ Estatuto Orgánico de Gestión Organizacional por Procesos determina que la misión de la Fuerza Terrestre (F.T.) es la de "desarrollar el poder militar terrestre, preparando, entrenando y equipando al personal militar, mejorando su capacidad operativa de forma permanente y en todo el territorio nacional para alcanzar los objetivos derivados de la planificación estratégica militar, a fin de coadyuvar en forma conjunta a la defensa de la soberanía e integridad territorial, contribuir en el ámbito interno y en el desarrollo de la nación", con el objeto de encontrarse en condiciones de ser empleada por el Comando Conjunto de las Fuerzas Armadas (CC.FF.AA.), ante cualquier escenario.

En este sentido, la F.T cuenta con el Comando de Operaciones Terrestres (C.O.T.), el cual es el responsable de la instrucción y entrenamiento, así como de la gestión de las operaciones que se disponen dentro de su competencia, como sigue:

- La administración de las capacidades militares;

- La instrucción, el entrenamiento y cultura física;

- El apoyo aéreo a las operaciones militares y a otras instituciones del Estado;

- La identificación y neutralización de material explosivo, desminado humanitario, misiones de paz;

- La evaluación y certificación de la instrucción y entrenamiento, $\mathrm{y}$

- El seguimiento de las operaciones militares que el CC.FF. AA. planifica y conduce a través de los Comandos Operacionales.

Bajo este marco normativo y conceptual, la presente investigación contribuye al proyecto del proceso de transformación ${ }^{1}$ emprendido por el Ejército del Ecuador a partir del año 2019 (Dirección de Transformación y Desarrollo Militar, 2020, pág. 10), el cual considera diez (10) líneas de transformación, siendo una de ellas, el entrenamiento.

La investigación presenta un diagnóstico de la instrucción y entrenamiento respecto a efectivos entrenados, pistas de entrenamiento, los promedios del acondicionamiento físico por tablas y grados de todo el personal militar y la infraestructura y centros de instrucción disponibles; $y$, en función de los resultados presentados, se establecieron estrategias y objetivos con sus respectivos indicadores, todos ellos distribuidos en períodos de tiempo en los cuales se considera un Tiempo 0 (T0) de transición con una duración de dos años: T1 de fortalecimiento, T2 de modernización y T3 de innovación; estos últimos con una duración de cuatro años en un horizonte temporal proyectado al año 2033 y que atiende a la visión del futuro institucional.

\footnotetext{
"La transformación es un proceso continuo que, a través de una actitud innovadora, se anticipa a las amenazas futuras, desarrollando nuevas competencias y capacidades para aumentar la eficiencia del Ejército, cumplir nuevas misiones y contribuir a la protección del Estado; implica un cambio profundo y disruptivo en los conceptos de cómo realizar las operaciones, en la organización, en la dimensión humana y en el material y equipo militar, que son influenciados directamente por la tecnología o el escenario" (DTDM, 2020).

${ }^{2}$ Cultura Física corresponde al acondicionamiento físico, deportes y recreación.
}

Las estrategias y objetivos planteados en función del tiempo establecido, permitirán contribuir al desarrollo de una fuerza con las capacidades necesarias que pueda adaptarse a los nuevos desafíos que garanticen el cumplimiento de las misiones del ejército.

\section{Conceptualización de LDT "Entrenamiento"}

Como parte de la investigación y argumentación para poder establecer la conceptualización de la LDT se comparó la información con los modelos de entrenamiento y misiones que cumplen los ejércitos de los siguientes países: Estados Unidos (Rodríguez, 2020), España (Guaytarilla, 2020), Brasil (Martínez, 2020), Chile (Calero, 2020) y Colombia (Ponce, 2020); de donde se propone el siguiente concepto:

Mejorar el actual modelo de instrucción, entrenamiento ${ }^{2}$, cultura física y entrenamiento físico militar individual y colectivo a través de modernas metodologías, y la implementación de centros de entrenamiento que permitan incrementar el alistamiento operacional de las unidades militares para hacer frente a los escenarios actuales y futuros.

\section{Descripción de la línea base LDT "Entrenamiento" \\ 1. Línea base del modelo de entrenamiento}

Para realizar el análisis de la línea base del modelo de entrenamiento que gestiona y es de responsabilidad del COT, se consideró al personal profesional entrenado $\mathrm{y}$ las pistas de entrenamiento que disponen las unidades del ejército y el estado y condiciones en que se encuentran; lo cual se detalla a continuación:

a. Efectivos entrenados: se tomó los datos del personal militar profesional entrenado desde el año 2016 al 2019, considerando la relación entre el efectivo total del ejército con el personal profesional entrenado.

\section{Tabla 1}

Efectivos entrenados

\begin{tabular}{lcccc}
\hline AÑO & $\mathbf{2 0 1 6}$ & $\mathbf{2 0 1 7}$ & $\mathbf{2 0 1 8}$ & $\mathbf{2 0 1 9}$ \\
EFECTIVO & 24933 & 25022 & 25184 & 24593 \\
PERS. PROF.ENTRENADO & 14992 & 15384 & 16194 & 16394 \\
$\%$ & $60,1 \%$ & $61,4 \%$ & $64,3 \%$ & $66,6 \%$ \\
MEDIA (LINEA BASE) & \multicolumn{5}{c}{$63 \%$} \\
\hline
\end{tabular}

Datos del informe de Gestión del C.G.E 2018, 2019 y D.G.T.H.E

Con base en la información y de acuerdo al análisis se determina que la línea base de los efectivos entrenados es del $63 \%$.

b. Pistas de entrenamiento: Se tomó en consideración las pistas fundamentales existentes:

El estudio considera a los Fuertes Militares y las 53 unidades tipo Batallón/Grupo; dicha información se detalla en la Tabla 2. 
Tabla 2

Pistas militares existentes y estado de las mismas

\begin{tabular}{lcccc}
\hline PISTAS & $\begin{array}{c}\text { BUEN } \\
\text { ESTADO }\end{array}$ & $\begin{array}{c}\text { REGULAR } \\
\text { ESTADO }\end{array}$ & $\begin{array}{c}\text { MAL } \\
\text { ESTADO }\end{array}$ & TOTAL \\
INDV. COMBATE & 3 & 10 & 9 & 22 \\
MILITAR & 1 & 7 & 0 & 8 \\
MUSCULACIÓN & 14 & 27 & 6 & 47 \\
CABOS & 2 & 5 & 3 & 10 \\
PENTATLON & 7 & 10 & 10 & 27 \\
PISCINA & 26 & 9 & 0 & 35 \\
CABO VERTICAL & 23 & 20 & 4 & 47 \\
TOTAL & 76 & 88 & 32 & 196 \\
PORCENTAJE & $39 \%$ & $45 \%$ & $16 \%$ & $100 \%$ \\
\hline Datos obtenidos del Comando de Operaciones Terrestre & &
\end{tabular}

\section{Línea base del acondicionamiento físico}

Para determinar la línea base en el parámetro de cultura física (acondicionamiento físico, deportes y recreación (Andrade, 2014, págs. 18-19)) se tomó en consideración el acondicionamiento físico por tablas de pruebas físicas de acuerdo a la edad del evaluado y grados militares de oficiales y tropa, así como el promedio por semestres de los tres últimos años.
No se disponen de datos de la evaluación de la preparación física militar para el empleo en las operaciones, así como tampoco las especificidades de pruebas físicas para cada una de las armas, servicios y empleo como equipos de combate, secciones, pelotones, compañías/ baterías/ escuadrones, batallones/ grupos, brigadas y divisiones.

Tabla 3

Promedio por tablas y grados (oficiales) - Datos obtenidos del Comando de Operaciones Terrestre

\begin{tabular}{|c|c|c|c|c|c|c|c|c|c|c|c|}
\hline & DESDE & HASTA & GRAD & GRAB & CRNL & TCRN & MAYO & CAPT & TNTE & SUBT & $\underset{\text { TABLAS }}{\mathbf{x}}$ \\
\hline $\begin{array}{c}\text { TABLA } \\
1\end{array}$ & - & 24 a $11 \mathrm{~m}$ & & & & & & & & 19,26 & 19,26 \\
\hline $\begin{array}{c}\text { TABLA } \\
2\end{array}$ & 25 años & 27 a $11 \mathrm{~m}$ & & & & & & & 19,4 & 19,31 & 19,36 \\
\hline $\begin{array}{c}\text { TABLA } \\
3\end{array}$ & 28 años & 30 a $11 \mathrm{~m}$ & & & & & & 19,04 & 19,03 & 19,43 & 19,17 \\
\hline $\begin{array}{c}\text { TABLA } \\
4\end{array}$ & 31 años & 23 a $11 \mathrm{~m}$ & & & & & & 19,23 & 18,84 & & 19,04 \\
\hline $\begin{array}{c}\text { TABLA } \\
5\end{array}$ & 34 años & 36 a $11 \mathrm{~m}$ & & & & & 20 & 18,98 & 17,58 & & 18,85 \\
\hline $\begin{array}{l}\text { TABLA } \\
6\end{array}$ & 37 años & 39 a $11 \mathrm{~m}$ & & & & & 17,86 & 18,99 & & & 18,43 \\
\hline $\begin{array}{c}\text { TABLA } \\
7\end{array}$ & 40 años & 42 a $11 \mathrm{~m}$ & & & & & 18,87 & 16,77 & & & 17,82 \\
\hline $\begin{array}{c}\text { TABLA } \\
8\end{array}$ & 43 años & 45 a $11 m$ & & & & 16,98 & 19,04 & 13,21 & & & 16,41 \\
\hline $\begin{array}{c}\text { TABLA } \\
9\end{array}$ & 46 años & 48 a $11 m$ & & & & 18,65 & 17,33 & & & & 17,99 \\
\hline $\begin{array}{c}\text { TABLA } \\
10\end{array}$ & 49 años & 51 a $11 \mathrm{~m}$ & & & 15,56 & 17,61 & 17,78 & & & & 16,98 \\
\hline TABLA & 52 años & 54 a $11 m$ & & 20 & 18,95 & 18,5 & 13,33 & & & & 17,70 \\
\hline $\begin{array}{c}\text { TABLA } \\
12\end{array}$ & 55 años & 57 a $11 m$ & 20 & 18,67 & 18,11 & 20 & & & & & 19,20 \\
\hline $\begin{array}{c}\text { TABLA } \\
13\end{array}$ & 58 años & $\begin{array}{c}\text { en } \\
\text { adelante }\end{array}$ & 20 & 20 & 16,96 & 13,13 & & & & & 17,52 \\
\hline \multicolumn{3}{|c|}{ PROMEDIO POR GRADOS } & 20,00 & 19,56 & 17,40 & 17,48 & 17,74 & 17,70 & 18,71 & 19,33 & 18,49 \\
\hline
\end{tabular}


Tabla 4

Promedio por tablas y grados (tropa) - Datos obtenidos del Comando de Operaciones Terrestre

\begin{tabular}{|c|c|c|c|c|c|c|c|c|c|c|c|}
\hline & DESDE & HASTA & SUBM & SUBP & SUBS & SGOP & SGOS & CBOP & cBOS & SLDO & $\underset{\text { TABLAS }}{\mathbf{X}}$ \\
\hline $\begin{array}{c}\text { TABLA } \\
1\end{array}$ & - & $\begin{array}{l}24 \text { a } 11 \\
m\end{array}$ & & & & & & & 18,69 & 18,87 & 18,78 \\
\hline $\begin{array}{c}\text { TABLA } \\
2\end{array}$ & 25 años & $\begin{array}{l}27 \text { a } 11 \\
m\end{array}$ & & & & & & & 18,85 & 19,2 & 19,03 \\
\hline $\begin{array}{c}\text { TABLA } \\
3\end{array}$ & 28 años & $\begin{array}{l}30 \mathrm{a} \\
11 \mathrm{~m}\end{array}$ & & & & & & 18,7 & 19,02 & 19,19 & 18,97 \\
\hline $\begin{array}{c}\text { TABLA } \\
4\end{array}$ & 31 años & $\begin{array}{l}23 a \\
11 m\end{array}$ & & & & & 16,61 & 18,77 & 18,93 & 19,49 & 18,45 \\
\hline $\begin{array}{c}\text { TABLA } \\
5\end{array}$ & 34 años & $\begin{array}{l}36 \mathrm{a} \\
11 \mathrm{~m}\end{array}$ & & & & & 18,4 & 18,72 & 18,87 & 19,31 & 18,83 \\
\hline $\begin{array}{c}\text { TABLA } \\
6\end{array}$ & 37 años & $\begin{array}{l}39 \mathrm{a} \\
11 \mathrm{~m}\end{array}$ & & & & 19,67 & 18,34 & 18,28 & 18,64 & & 18,73 \\
\hline $\begin{array}{c}\text { TABLA } \\
7\end{array}$ & 40 años & $\begin{array}{l}42 \mathrm{a} \\
11 \mathrm{~m}\end{array}$ & & & & 18,47 & 18,35 & 18,24 & 17,46 & & 18,13 \\
\hline $\begin{array}{c}\text { TABLA } \\
8\end{array}$ & 43 años & $\begin{array}{l}45 \mathrm{a} \\
11 \mathrm{~m}\end{array}$ & & & 17,98 & 17,98 & 18,36 & 16,13 & 19,65 & & 18,02 \\
\hline $\begin{array}{c}\text { TABLA } \\
9\end{array}$ & 46 años & $\begin{array}{l}48 \mathrm{a} \\
11 \mathrm{~m}\end{array}$ & & 20 & 18,53 & 18,09 & 19,51 & & & & 19,03 \\
\hline $\begin{array}{c}\text { TABLA } \\
10\end{array}$ & 49 aก̃os & $\begin{array}{l}51 \mathrm{a} \\
11 \mathrm{~m}\end{array}$ & 20 & 18,83 & 17,85 & 17.57 & 20 & & & & 18,85 \\
\hline $\begin{array}{c}\text { TABLA } \\
11\end{array}$ & 52 años & $\begin{array}{l}54 \mathrm{a} \\
11 \mathrm{~m}\end{array}$ & 20 & 19,17 & 17,84 & 14,26 & & & & & 17,82 \\
\hline $\begin{array}{c}\text { TABLA } \\
12\end{array}$ & 55 años & $\begin{array}{l}57 \mathrm{a} \\
11 \mathrm{~m}\end{array}$ & 20 & 20 & & & & & & & 20,00 \\
\hline $\begin{array}{c}\text { TABLA } \\
13\end{array}$ & 58 aก̃os & $\begin{array}{l}\text { en } \\
\text { adelante }\end{array}$ & & 20 & & & & & & & 20,00 \\
\hline \multicolumn{3}{|c|}{ PROMEDIO POR GRADOS } & 20,00 & 19,60 & 18,05 & 17,67 & 18,51 & 18,14 & 18,76 & 19,21 & 18,74 \\
\hline
\end{tabular}

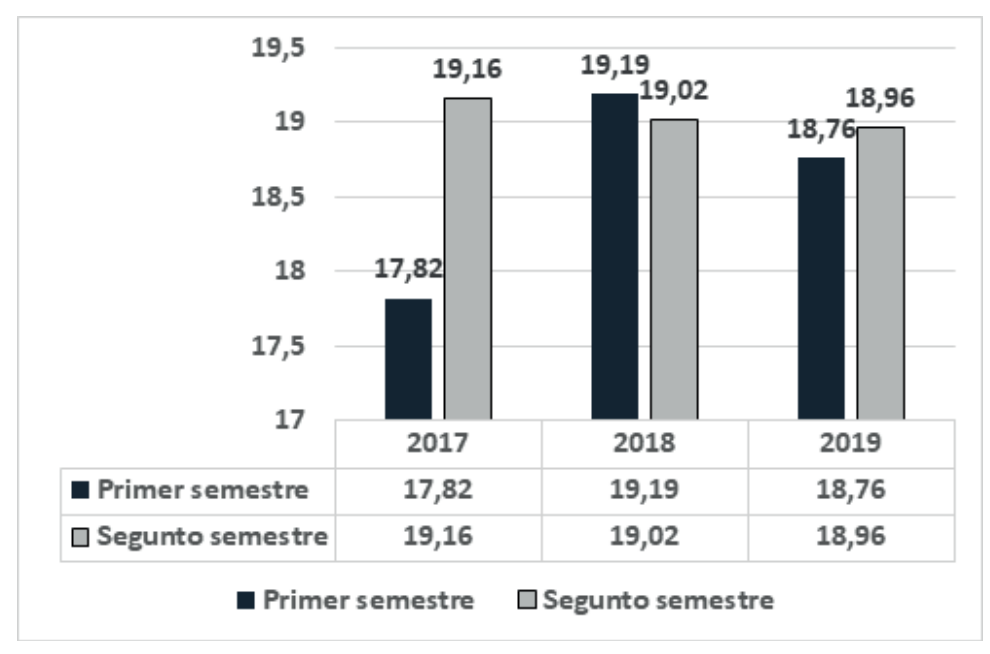

Figura 1. Promedio por semestres 
De la Figura 1 se desprende un promedio anual en los últimos tres años de 19.003

a. Acondicionamiento físico general. - Tomando en consideración la calificación de pruebas físicas por tablas se determina una línea base en pruebas físicas de oficiales es de 18.49; tropa, 18.74 y un promedio general de los últimos tres años es de 19.003.

b. Preparación física militar para el empleo en las operaciones. - El C.O.T. no cuenta con un parámetro para poder verificar la preparación física por armas, servicios, especialistas y por especialidad; por lo tanto, la línea base en este parámetro es $0 \%$.

\section{Centros de entrenamiento}

A fin de determinar la línea base en lo que corresponde a los centros de entrenamiento, se ha considerado la certificación de la instrucción y entrenamiento de las unidades del ejército desde el año 2017 al 2019, lo que puede determinarse mediante la certificación anual de unidades del ejército a nivel Batallón/Grupo, lo cual se presenta en el la Tabla 5 a continuación.

Tabla 5

Centros de entrenamiento

\begin{tabular}{lccc}
\hline AN̈O & $\begin{array}{c}\text { UNIDADES TIPO } \\
\text { BATALLON/GRUPO }\end{array}$ & $\begin{array}{c}\text { UNIDADES } \\
\text { EVALUADAS }\end{array}$ & $\begin{array}{c}\text { PORCENTAJE } \\
2017\end{array}$ \\
53 & 18 & $33,96 \%$ \\
2018 & 53 & 18 & $33,96 \%$ \\
2019 & 53 & 17 & $32,07 \%$ \\
& & Promedio & $33,33 \%$
\end{tabular}

Datos del C.G.E 2019 (C.I.E.C.E - C.E.C.S.E-COT)

Con base en la existencia y capacidad instalada de los centros de certificación con relación al número de unidades tipo Batallón / Grupo, la línea base se determina que el 33,3\% de las unidades del ejército se certifican anualmente. egresados y alumnos de los últimos cursos de la Academia de Defensa Militar Conjunta, Oficiales Superiores y en representación de la tropa, los Suboficiales del Ejército. En total se realizaron 65 encuestas, de acuerdo al detalle plasmado en la Figura 2 a continuación.

\section{Relaciones entre líneas de transformación y gráfico de convergencia}

Para realizar el gráfico de convergencia, se creyó pertinente realizar una encuesta dirigida a personal profesional de las tres ramas de las FF.AA. como son:

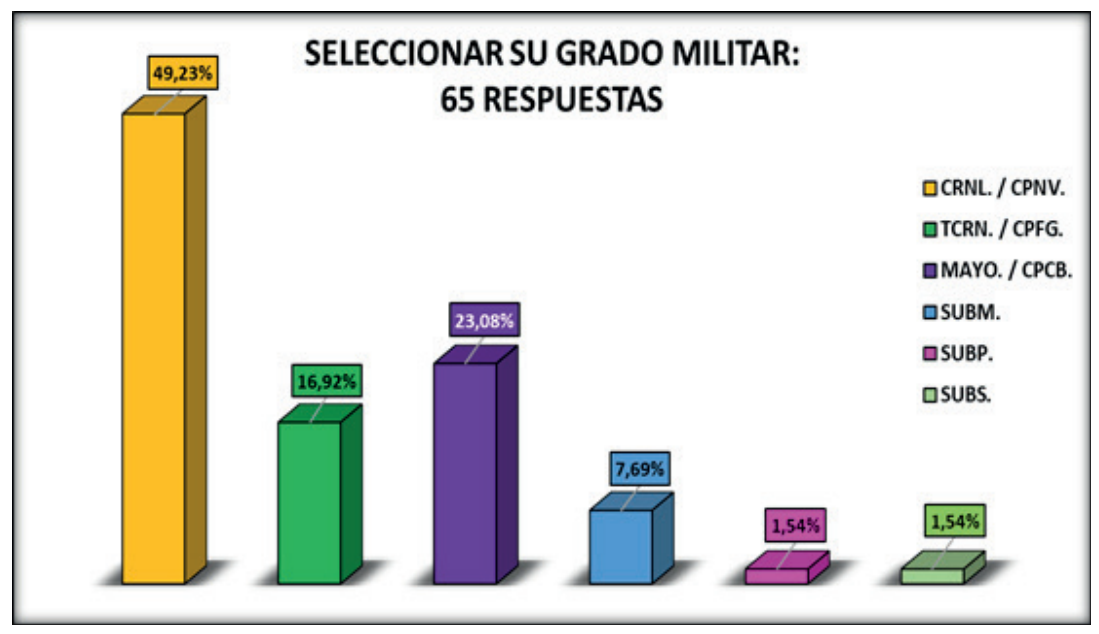

Figura 2. Resultado de las encuestas aplicadas 
Para la correcta solución de la encuesta, se proporcionó a los encuestados la información referente a los significados de los conceptos de las 10 líneas de transformación que actualmente se maneja, de la siguiente manera:

\section{Entrenamiento}

Son todas las actividades tendientes a la preparación del personal militar tanto en forma individual como colectiva (unidades militares), con el fin de conseguir las capacidades necesarias para el cumplimiento de todo tipo de misiones, por lo que se deberá mejorar los actuales modelos de entrenamiento y acondicionamiento físico con metodologías modernas y la implementación de centros de entrenamiento, que incrementen el alistamiento operacional de las unidades y así hacer frente a los escenarios actuales y futuros.

\section{Doctrina}

Conjunto de saberes específicos y actualizados que describen los principios y conceptos fundamentales del empleo del ejército, los cuales orientan el cumplimiento eficiente de las misiones encomendadas y permiten enfrentar amenazas y desafíos actuales y futuros.

\section{Organización}

Constituye la manera en la cual una fuerza o unidad está estructurada con el fin de cumplir las misiones y los objetivos emanados del Escalón Superior, la misma que debe ser flexible y adaptarse a las situaciones que se presenten.

\section{Desarrollo tecnológico}

Es todo tipo de actividades relacionadas con la formulación de proyectos de investigación, desarrollo e innovación de sistemas, plataformas, armamento, equipo y vestuario con el aporte de universidades, centros de investigación y empresas tanto nacionales y extranjeras, para incrementar las capacidades de los sistemas operativos del campo de batalla y lograr un ejército multipropósito.

\section{Educación}

Es un proceso de transferencia multidireccional de conocimiento, habilidades, creencias y valores, por lo que se debe implementar un cambio significativo en este proceso de enseñanza - aprendizaje por medio de la innovación pedagógica y el uso de tecnología, para aumentar el pensamiento crítico y creativo del personal militar y prepararlo para enfrentar las complejidades de los nuevos escenarios.

\section{Gestión humana}

Son todas las acciones que se realizan para administrar el talento humano, por lo que se debe efectuar mejoras sustanciales en los procesos relacionados con el personal del ejército: obtención de personal, incentivos, desempeño, evaluación, capacitación, salud, manejo, clima laboral, desarrollo y bienestar de personal.

\section{Cultura institucional}

Es un sistema de significados compartidos por todos los miembros de una organización $\mathrm{y}$, en este sentido se deben generar cambios profundos en los valores, creencias, hábitos, actitudes y tradiciones de los miembros del ejército que fomenten una innovación significativa en la forma de hacer las cosas dentro de la institución, razón por la cual es base de la transformación y depende del liderazgo ejercido en todos los niveles de mando.

\section{Sostenimiento institucional}

Constituye la provisión de recursos, por lo que se debe modernizar las actividades de obtención, distribución y mantenimiento de los recursos materiales y armamento requeridos para el empleo efectivo de la Fuerza, a través de estructuras, sistemas, planes, métodos y procedimientos innovadores, que aseguren las operaciones en los escenarios de actuación actuales y futuros, alcanzando eficiencia en el empleo de recursos y medios y, por ende, reduciendo el esfuerzo logístico.

\section{Despliegue e infraestructura}

Se refiere a la ubicación de las unidades militares en el país, que debe ser balanceada, armónica y efectiva, mejorando, además, su infraestructura física e instalaciones para que sean más eficientes y resilientes. Adicionalmente, se debe implementar o modernizar su infraestructura tecnológica para alcanzar la hiperconectividad a través de planes y proyectos técnicamente concebidos.

\section{Marco legal}

Se refiere al contenido de leyes y normas que regulan la carrera y conducta del personal militar, así como de aquellas que regulan las misiones encomendadas a las FF. AA. en los ámbitos de la defensa y seguridad integral, incluyendo el orden público y la gestión de riesgos. (Dirección de Transformación y Desarrollo Militar, 2020, págs. 14,16).

Las preguntas de la encuesta se centraron directamente en los criterios de relacionamiento del entrenamiento (alto, medio y bajo) en relación con cada una de las otras líneas de transformación con su respectiva justificación. La tabulación de los resultados se presenta a continuación:

Los resultados de las encuestas nos indican que, el criterio de los oficiales superiores y suboficiales manifiesta que el entrenamiento tiene un relacionamiento alto con ocho (8) ejes de transformación, a excepción del eje de sostenimiento institucional cuyo relacionamiento es medio; por otra parte, no existe una LDT que tenga un relacionamiento bajo con el entrenamiento. Esto se explica debido a que el entrenamiento es la base fundamental del alistamiento operacional, razón de ser de nuestra institución y uno de los pilares fundamentales para la formación del talento humano, que al final determinará el cumplimiento de los objetivos de una fuerza multimisión con los más altos estándares de eficiencia y acorde a las exigencias actuales y futuras del Ejército Ecuatoriano.

De igual manera, se requirió en la encuesta la justificación de los criterios de relacionamiento del entrenamiento con las otras nueve líneas de transformación, lográndose obtener 585 respuestas, las cuales se sintetizan en la siguiente Matriz de Impacto: 


\begin{tabular}{|c|c|}
\hline DOCTRINA & IMPACTO ALTO \\
\hline \multicolumn{2}{|c|}{$\begin{array}{l}\text { La Doctrina es la guía para cumplir las operaciones, por lo que constituye la } \\
\text { base para establecer los objetivos del entrenamiento. } \\
\text { Al ser la guía para el cumplimiento de las operaciones, constituye la base } \\
\text { para establecer los objetivos del entrenamiento. } \\
\text { El entrenamiento puede retroalimentar a la doctrina }\end{array}$} \\
\hline ORGANIZACIÓN & IMPACTO ALTO \\
\hline \multicolumn{2}{|c|}{$\begin{array}{l}\text { Depende de la estructura organizacional y sus capacidades, para que se } \\
\text { diseñe una fuerza bien entrenada que permita el cumplimiento de las } \\
\text { misiones. } \\
\text { Permite desarrollar destrezas y habilidades colectivas mediante el } \\
\text { entrenamiento }\end{array}$} \\
\hline EDUCACIÓN & IMPACTO ALTO \\
\hline \multicolumn{2}{|c|}{$\begin{array}{l}\text { El entrenamiento tiene mucho que ver con los procesos de enseñanza } \\
\text { aprendizaje adecuados para enfrentar los nuevos escenarios, por lo que la } \\
\text { educación viene a ser la base fundamental del entrenamiento. Además, } \\
\text { debemos contar con instructores y preparadores físicos altamente } \\
\text { calificados. }\end{array}$} \\
\hline GESTIÓN HUMANA & IMPACTO ALTO \\
\hline \multicolumn{2}{|c|}{$\begin{array}{l}\text { El talento humano es el principal insumo en el entrenamiento militar, ya que } \\
\text { un soldado calificado, formado en valores y con las cualidades y habilidades } \\
\text { necesarias, es apto para el entrenamiento. } \\
\text { La gestión humana satisface las necesidades del personal para que este se } \\
\text { enfoque totalmente en las exigencias del entrenamiento. }\end{array}$} \\
\hline MARCO LEGAL & IMPACTO ALTO \\
\hline \multicolumn{2}{|c|}{$\begin{array}{l}\text { El entrenamiento va a depender directamente de la base legal que permita la } \\
\text { forma de actuación del miembro del ejército en las diferentes misiones } \\
\text { asignadas y permitidas de acuerdo a la normativa legal vigente. } \\
\text { Constituye la base para accionar enmarcada en el derecho. Ej. Uso } \\
\text { proqresivo de la fuerza. }\end{array}$} \\
\hline
\end{tabular}

\begin{tabular}{|l|l|}
\hline \multicolumn{1}{|c|}{ DESPLIEGUE E } & \multicolumn{1}{c|}{ IMPACTO ALTO } \\
INFRAESTRUCTURA & \\
\hline $\begin{array}{l}\text { Si se desea un nivel de entrenamiento y exigencia máximo, las instalaciones } \\
\text { requeridas deben cumplir con los estándares mínimos indispensables. } \\
\text { Los centros de entrenamiento son importantes porque se instruye a todo el } \\
\text { personal militar. } \\
\text { El entrenamiento debe ser ejecutado en función de las competencias de cada } \\
\text { militar, por lo que la Fuerza debería disponer de una infraestructura } \\
\text { adecuada para el entrenamiento común y específico para las operaciones en } \\
\text { Costa, Sierra y Oriente. }\end{array}$ \\
\hline \multicolumn{2}{|c|}{ DESARROLLO } \\
\multicolumn{2}{|c|}{ IMPACTO ALTO } \\
\hline $\begin{array}{l}\text { El entrenamiento se complementa con recursos tecnológicos a fin de } \\
\text { alcanzar estándares de calidad y eficiencia. } \\
\text { El empleo de la tecnología permite reducir costos para el entrenamiento, } \\
\text { disminuir tiempos, optimizar recursos evitando su desgaste. A mayor } \\
\text { desarrollo tecnológico mejor resultado en el entrenamiento. }\end{array}$ \\
\hline
\end{tabular}




\begin{tabular}{|l|l|}
\hline \multicolumn{1}{|c|}{ CULTURA } & \multicolumn{1}{|c|}{ IMPACTO ALTO } \\
INSTITUCIONAL & \\
\hline $\begin{array}{l}\text { La cultura organizacional determina las características de la institución, ya } \\
\text { que en esta se refleja su historia, y tradiciones. } \\
\text { El entrenamiento fortalece la práctica de valores institucionales y promueve } \\
\text { el compromiso de sus integrantes. } \\
\text { Con el entrenamiento se logra incrementar la forma de actuar del recurso } \\
\text { humano y ello a su vez fortalece la cultura organizacional. }\end{array}$ \\
\hline \multicolumn{2}{|c|}{ SOSTENIMIENTO } \\
INSTITUCIONAL
\end{tabular}

Nota. Resultado del análisis de las encuestas. (Equipo de análisis de las LDT "ENTRENAMIENTO", 2020)

De toda la información descrita, el Gráfico de Convergencia resulta:

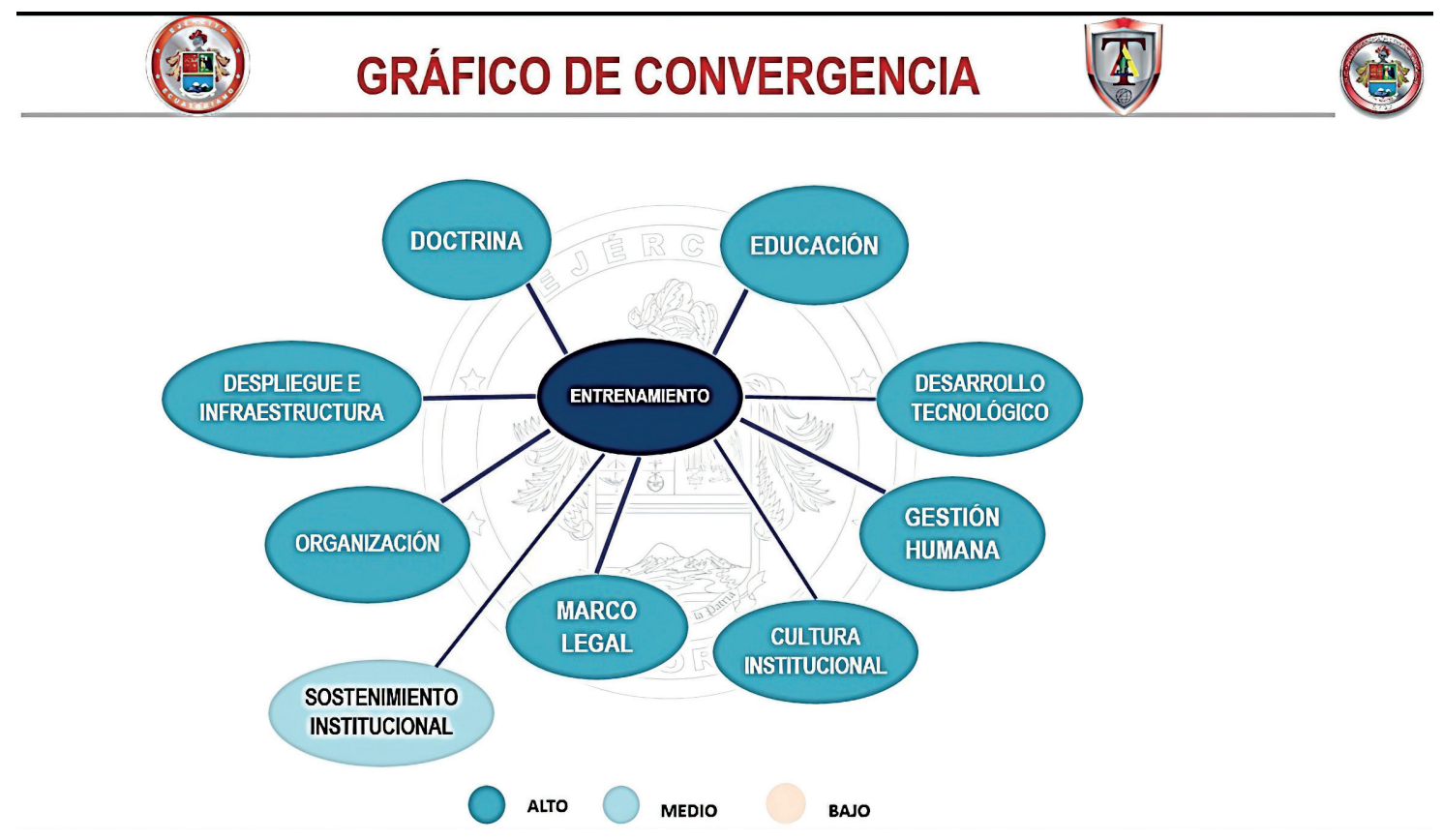

Figura 3. Relacionamiento de las $L D T$

III. Desarrollo de objetivos, estrategias e indicadores para cada objetivo en cada tiempo

Con base en la conceptualización de la LDT entrenamiento que indica: "Mejorar el actual modelo de instrucción, entrenamiento, cultura física y entrenamiento físico militar, individual y colectivo, a través de modernas metodologías y la implementación de centros de entrenamiento, que permitan incrementar el alistamiento operacional de las unidades militares para hacer frente a los escenarios actuales y futuros". Se establecen tres áreas generales que conforman el entrenamiento:

- Modelos de entrenamiento 
- Cultura Física (CF) y Entrenamiento Físico Militar (EFM)

- Centros de Entrenamiento

Estas tres áreas generales de la LDT "Entrenamiento", estarán enfocadas en los cuatro tiempos que determina la transformación del Ejército con sus respectivos objetivos y estrategias.

1. Objetivo, estrategia e indicador tiempo to (2019 - 2021)

a. Determinar el nivel de liderazgo existente en los diferentes niveles de mando en la actualidad.

La estrategia se plantea: evaluando a través de la plataforma SIVEC $^{3}$ y aplicando encuestas (preguntas cerradas) utilizando medios virtuales.

Los indicadores serán: porcentaje de personal militar que alcanzó la nota igual o superior a 14/20 y porcentaje de respuestas correctas, respectivamente.

b. Determinar el modelo de entrenamiento del personal militar que permita mantener un óptimo alistamiento operacional, orientado al cumplimiento de las misiones.

La estrategia se plantea: Evaluando el Plan de Gestión Operacional (PGO) actual y actualizando el PGO.

El indicador será: Resultado del PGO evaluado y actualizado.

c. Determinar las necesidades de los simuladores físicos y virtuales, para el entrenamiento en las diferentes especialidades en concordancia con los escenarios actuales y futuros.

La estrategia se plantea: estableciendo la situación actual de los simuladores existentes y estableciendo los requerimientos de simuladores a las unidades militares (divisiones).

Los indicadores serán: número de simuladores operables / número de simuladores existentes, y número de requerimientos de simuladores de las unidades / número de simuladores existentes, respectivamente.

\section{Objetivo, estrategia e indicador. Tiempo T1 (2021} - 2025)

a. Desarrollar líderes a través de programas de fortalecimiento del liderazgo con nuevos sistemas de desempeño profesional,

La estrategia se plantea: mejorando el nivel de liderazgo en los diferentes niveles de mando mediante la implementación de cursos virtuales a través de la plataforma SIVEC, seminarios, etc.

El indicador será: porcentaje de personal militar que alcanzó la nota igual o superior a 17/20.

b. Ejecutar el modelo de entrenamiento militar en la defensa del territorio nacional y el ámbito interno que permita hacer frente a los escenarios actuales y futuros.

${ }^{3}$ SIVEC: Es el Sistema Virtual de Educación Continua que funciona en una plataforma administrada por la Universidad de Fuerzas Armadas ESPE.
La estrategia se plantea: Cumpliendo con el PGO actualizado donde consta el modelo de instrucción y entrenamiento.

Los indicadores serán: número de personal profesional entrenado / número de personal profesional planificado, y número de personal instruido $\left(\mathrm{SCMV}^{4}\right) /$ número de personal planificado.

c. Gestionar proyectos para implementación de simuladores en todas las armas y servicios, para la reducción del gasto.

La estrategia se plantea: disponiendo a los sistemas de armas, servicios y especialistas propongan proyectos de simuladores donde se debe incluir el presupuesto requerido.

El indicador será: presupuesto requerido para la implementación de proyectos / presupuesto disponible en la fuerza.

\section{Objetivo, estrategia o indicador. Tiempo 2 (2025} - 2029)

a. Implantar un software que permita realizar evaluaciones periódicas de sus líderes, con análisis de sus destrezas, sus hábitos de trabajo y sus objetivos profesionales e individuales, los cuales facilitarán la toma de decisiones más inteligentes.

Las estrategias se plantean: Desarrollando el software con el apoyo de la Universidad de FF.AA. y desarrollando el software con el apoyo de empresa privada o la academia (universidades).

Los indicadores serán: número de ensayos positivos / número de ensayos fallidos y disponer del software desarrollado, respectivamente.

b. Mejorar el modelo de entrenamiento militar en la defensa del territorio nacional y en el ámbito interno que permita hacer frente a los escenarios actuales y futuros.

La estrategia se plantea: evaluando e innovando el PGO.

El indicador será: porcentaje de cumplimiento del PGO.

c. Ejecutar proyectos e implementar simuladores con realidad virtual, $4 \mathrm{G}$ y realidad dirigida, tanto para tiro, como para otros tipos de entrenamiento.

La estrategia se plantea: designando equipos multidisciplinarios para el desarrollo de los proyectos.

El indicador será: disponer de simuladores de realidad virtual.

\section{Objetivo, estrategia o indicador. Tiempo 3 (2029} - 2033)

a. Disponer de líderes mentores que permitan desarrollar e influir en los futuros líderes de la institución, motivadores de su unidad, con libertad de acción para la conducción de sus unidades y multiplicador de la cohesión institucional.

La estrategia se plantea: evaluando en las diferentes unidades el desempeño del personal militar 
profesional en los diferentes niveles por parte de los comandos de las unidades mediante el software desarrollado.

El indicador será: número de escenarios desarrollados satisfactoriamente por el personal militar profesional / número de escenarios planteados en la evaluación.

b. Disponer del modelo de entrenamiento militar en la defensa del territorio nacional y en el ámbito interno que permita hacer frente a los escenarios actuales y futuros.

La estrategia se plantea: evaluando e innovando el PGO.

El indicador será: porcentaje de cumplimiento del PGO.

c. Disponer de tecnología de punta que permita dar el soporte para modelar escenarios virtuales de entrenamiento cambiantes para el empleo individual y con tropas en cada nivel de conducción, permitiendo así desarrollar una mejor capacidad de liderazgo y aplicación de la doctrina en el entrenamiento.

La estrategia se plantea: desarrollando, a través del CIMSE$^{5}$, alianzas estratégicas y cooperación internacional, para modelar escenarios virtuales de entrenamiento cambiantes para el empleo individual y con tropas en cada nivel de conducción.

Los indicadores serán: número de proyectos implementados y porcentaje de personal militar profesional entrenado.

\section{Objetivos de Cultura Física (CF) y Entrenamiento Físico Militar (EFM)}

a. Objetivo, estrategia o indicador. Tiempo T0 (2019-2021)

1) Establecer a la cultura física como una cualidad fundamental del militar, orientado a incrementar las destrezas físicas, salvaguardando su salud y adecuada alimentación para alcanzar su óptimo desempeño en el cumplimiento de la misión y tareas asignadas.

Las estrategias se plantean: Incrementando estímulos a la excelencia física para el personal que destaque en las evaluaciones formales (marcas); realizando campañas comunicacionales para concienciar al personal sobre la importancia de la actividad física y el entrenamiento militar y receptando anualmente las medidas antropométricas ${ }^{6}$ del personal militar para conocer los porcentajes de masa muscular, ósea y grasa corporales y realizar la planificación del EFM.

Los indicadores serán: número de encomios simples por grado / año / número de grados (18); número de campañas ejecutadas / número de campañas planificadas; $y$, número de medidas antropométricas

${ }^{4}$ SCMV es el Servicio Cívico Militar Voluntario

${ }_{5}^{5}$ CIMSE es el Centro de Instrucción, Monitoreo y Simulación del Ejército. ejecutadas / número de medidas antropométricas planificadas, respectivamente.

2) Cambiar la concepción del entrenamiento físico tradicional por el entrenamiento físico funcional para desarrollar cualidades físicas básicas mediante la combinación de las modalidades de entrenamiento que permitan al personal militar alcanzar su más alto rendimiento físico. (Haddock, Poston, Heinrich, Jahnke, \& Jitnarin, 2016).

Las estrategias se plantean: Coordinando con la Carrera de Cultura Física de la UFA-ESPE la realización de cursos permanentes de actualización para el personal de Oficiales y Voluntarios que desempeñan funciones de esta especialidad en las unidades militares; manteniendo los cupos de ingreso a la especialidad de Cultura Física en la UFA-ESPE (cursos intensivos de 1 año); ejecutando cursos para monitores y auxiliares de Cultura Física en la UFA-ESPE por el lapso de 3 meses; gestionando pasantías/becas al exterior para oficiales y voluntarios destacados en la especialidad; $\mathrm{y}$, actualizando planes y programas de CF y EFM de acuerdo a la tendencia actual. Los indicadores serán: número de cursos de actualización ejecutados / número de cursos de actualización planificados; número de cupos de ingresos anuales / número de cupos anuales establecidos; número cursos ejecutados / número de cursos planificados; y número de pasantías/becas gestionadas por año.

3) Organizar la especialidad de CF de la F.T. para alcanzar los objetivos del entrenamiento (LDT).

La estrategia se plantea: fortaleciendo la organización de la especialidad de CF del ejército. El indicador será: número de integrantes disponibles / número de integrantes requerido.

b. Objetivo, estrategia o indicador. Tiempo T1 (2021 - 2025)

1) Contribuir a determinar una base legal que establezca las directrices fundamentales que sustenten la preparación, optimización y eficiencia de la cultura física $(\mathrm{CF})$ y el entrenamiento físico militar (EFM).

La estrategia se plantea: Organizando a través del CEDMT comisiones de Investigación para la elaboración del reglamento o manual de CF y EFM del ejército.

El indicador será: Reglamento de Cultura Física del Ejército aprobado.

2) Planificar y ejecutar la CF y el EFM para desarrollar las capacidades físicas considerando la naturaleza de las unidades militares y sus misiones. Las estrategias se plantean: diseñando e

${ }^{6}$ Los datos que arrojan las medidas antropométricas son más reales y confiables que los determinados por la simple relación peso - estatura

${ }^{7} \mathrm{El}$ entrenamiento funcional significa entrenar con un propósito, un entrenamiento para que sirva. Es una metodología de entrenamiento que se basa en realizar ejercicios y movimientos que nos ayuden a desempeñar tareas funcionales 
implementando programas de entrenamiento físico funcional ${ }^{7}$, conforme a la naturaleza de las unidades y a sus misiones: (operaciones convencionales, especiales y administrativas); Incluyendo en el EFM programas de auto defensa personal, artes marciales y técnicas de sumisión acordes con el uso progresivo de la fuerza; estableciendo un sistema de evaluación de las destrezas militares del Ejército (individual y colectiva); y, actualizando las normas de seguridad para la ejecución de la CF y el EFM El indicador será: porcentaje de unidades con al menos el 70 porcentaje de su planificación validada por el C.O.T.

3) Disponer de infraestructura, material y recursos para la ejecución de la planificación de la CF y EFM que permita cumplir con las exigencias del entrenamiento.

Las estrategias se plantean: planificando proyectos de recuperación de la infraestructura (gimnasios, pistas militares, etc.) para la CF y el EFM y la construcción de infraestructura para el entrenamiento funcional; $\mathrm{y}$, gestionando recursos para la adquisición de material y equipo para el cumplimiento de la planificación de la CF y del EFM.

Los indicadores serán: costo promedio de material y equipo acorde a naturaleza y misión de las unidades y porcentaje de asignación presupuestaria por parte del Estado para la ejecución de proyectos, respectivamente.

4) Incorporar a la ejecución de la CF y al EFM elementos tecnológicos que permitan la mejora de las capacidades físicas de la persona, así como, el control y evaluación de la evolución del entrenamiento.

La estrategia se plantea: realizando proyectos para implementar nuevas tecnologías aplicables al EFM. El indicador será: número de proyectos implementados.

c. Objetivo, estrategia o indicador. Tiempo T1 (2021 - 2025)

1) Mejorar el plan de CF y EFM de acuerdo a la naturaleza de las unidades militares y sus misiones. Las estrategias se plantean: capacitando permanentemente al personal perteneciente al Sistema de Cultura Física y asignando al personal especialista en Cultura Física en las unidades acorde a su especialidad.

El indicador será: porcentaje de participantes en los cursos que califican con un nivel de satisfacción alta $(18 / 20)$.

2) Planificar la creación de un Centro Alto Rendimiento Deportivo que permita la investigación y evaluación de la CF y el EFM de la F.T.

Las estrategias se plantean: generando proyectos para implementar un Centro de Alto Rendimiento que permitan realizar la evaluación e investigación de alto rendimiento para disponer de atletas de elite para las competencias militares internacionales y realizando alianzas estratégicas con la federación deportiva nacional; conformando una comunidad de investigadores en cultura física, medicina deportiva y nutrición que retroalimenten el manejo de la CF, el EFM y la preparación de atletas de elite, y diseñando programas de medicina deportiva que permitan mantener y mejorar las capacidades físicas del personal militar en general y de los deportistas de élite.

Los indicadores serán: porcentaje de asignación presupuestaria por parte del Estado respecto a la asignación para gasto de inversión de la F.T.; porcentaje de investigadores militares/civiles, y número de programas implementados / número de programas planificados, respectivamente.

d. Objetivo, estrategia o indicador. Tiempo T3 (2029 - 2033)

1) Disponer de la infraestructura y los recursos para innovar la planificación de la CF y el EFM que permita contar con una fuerza multimisión en óptimas condiciones.

Las estrategias se plantean: innovando continuamente la planificación y ejecución de la CF y el EFM y gestionando y asignando los recursos a las unidades militares para que la infraestructura se mantenga en óptimas condiciones para la práctica de la CF y del EFM.

Los indicadores serán: porcentaje de personal militar con evaluación de condición física superior a la base establecida; porcentaje de personal militar con evaluación de entrenamiento físico-militar superior a la base establecida, y porcentaje de la infraestructura disponible en las unidades militares en buen estado.

2) Disponer de centros de alto rendimiento con equipamiento deportivo y personal técnico certificado encargado de la investigación y evaluación de los planes y programas de CF, del EFM y de los deportistas de alto rendimiento.

La estrategia se plantea: implementando continuamente procesos para la optimización de la capacidad instalada del Centro de Alto Rendimiento Deportivo.

El indicador será: porcentaje de implementación de procesos del Centro de Alto Rendimiento del Ejército.

\section{Objetivos de los Centros de Entrenamiento}

a. Objetivo, estrategia o indicador. Tiempo T0 (2019-2021)

1) Determinar la situación en la que se encuentran los centros de entrenamiento del ejército.

La estrategia se plantea: evaluando mediante un plan a los centros de entrenamiento del ejército, por parte del C.O.T., de acuerdo a las amenazas y riesgos establecidos en el Capítulo V de la Política 
de la Defensa Nacional en infraestructura, material y equipo, personal disponible y capacitación.

El indicador será: porcentaje de operatividad y operabilidad del C.E.

2) Ejecutar el plan de evaluación en los centros de entrenamiento para la certificación periódica del entrenamiento del personal profesional de armas, servicios y especialistas.

Las estrategias se plantean: aplicando la Norma de Evaluación para las unidades tipo Batallón del Ejército. En las misiones establecidas en los planes de defensa externa y ámbito interno, de acuerdo a las amenazas y riesgos establecidos en el Capítulo V de la Política de la Defensa Nacional; evaluando a los Estados Mayores de las Brigadas y Planas Mayores de los Batallones, en coordinación con la A.G.E., mediante el CIMSE, y evaluando a los Estados Mayores de las Divisiones por parte del C.O.T., en coordinación con la A.G.E., mediante el CIMSE.

Los indicadores serán: porcentaje de unidades certificadas (18.5) / lo planificado; porcentaje de conocimiento y de conducción de las Planas Mayores y Estados Mayores (18.5), y porcentaje de conocimiento y de conducción de los Estados Mayores (18.5), respectivamente.

b. Objetivo, estrategia o indicador. Tiempo 1 (2021 - 2025)

1) Implementar en los centros el entrenamiento virtual en la toma de decisiones en los niveles de la conducción en la Defensa del Territorio Nacional y en el Ámbito Interno, que permita el entrenamiento y evaluación de comandantes y Estado/Plana Mayor a nivel Brigada y Batallón en los escenarios que tiene el país.

Las estrategias se plantean: gestionando y asignando los recursos económicos a los centros de entrenamiento para la construcción, equipamiento e implementación integral de módulos de entrenamiento y certificación virtual de armas y servicios, para las unidades del ejército, y desarrollando software, en coordinación con la UFA-ESPE, que permita la creación de escenarios virtuales para la capacitación y entrenamiento de los Estados Mayores y Planas Mayores, en la conducción de las operaciones militares de defensa externa y ámbito interno.

Los indicadores serán: porcentaje de recursos asignados para la implementación de módulos integrales de entrenamiento virtual; $y$, disponer del software.

c. Objetivo, estrategia o indicador. Tiempo 2 (2025 - 2029)

1) Modernizar el centro de entrenamiento virtual de toma de decisiones en los niveles de la conducción en la Defensa del Territorio Nacional y en el Ámbito Interno, que permita el entrenamiento y evaluación de comandantes y Estados Mayores y Planas Mayores a nivel Batallón y Brigada. La estrategia se plantea: gestionando y asignando los recursos económicos para modernizar los centros de entrenamiento virtual de la toma de decisiones. El indicador será: porcentaje de centros de entrenamiento virtual modernizados.

2) Desarrollar proyectos y presupuesto para la creación de centros de entrenamiento virtuales en cada división.

Las estrategias se plantean: gestionando y asignando los recursos económicos, para la construcción, equipamiento e implementación integral de módulos de entrenamiento y certificación virtual de armas y servicios en cada una de las Divisiones, y desarrollando software que permitan la creación de escenarios virtuales para la capacitación y entrenamiento de los Estados Mayores y Planas Mayores, en la conducción de las operaciones militares de defensa externa y ámbito interno.

Los indicadores serán: número de Centros de Entrenamiento creados (División); y Software desarrollado, respectivamente.

\section{d. Objetivo, estrategia o indicador. Tiempo 3 (2029} - 2033)

1) Disponer de la sala de guerra virtual en el C.O.T. enlazada con centros de entrenamiento virtuales de toma de decisiones en cada uno de los comandos de División.

Las estrategias se plantean: gestionando y asignando los recursos al C.O.T. para disponer de la infraestructura y tecnología de punta para enlazar a los Centros de entrenamiento de las Divisiones, para el entrenamiento, y capacitando al personal de oficiales y voluntarios en la operación técnica y tecnológica de la sala de guerra virtual.

Los indicadores serán: disponer de la sala virtual de guerra en el COT; y, personal capacitado en los Centros de Entrenamiento virtual en los diferentes niveles. (Equipo de análisis de la LDT "Entrenamiento", 2020).

\section{Referencias}

Andrade, D. (2014). La Educación Física, el Deporte y la Recreación Conceptos, dinámicas recreacionales y métodos de enseñanza. Universidad de las Fuerzas Armadas ESPE.

Calero, C. E. (2020). Modelo de entrenamiento del Ejército de EE.UU. Santiago.

Dirección de Transformación y Desarrollo Militar. (2020). Modelo de Transformación del Ejército ecuatoriano, 14-16.

Equipo de análisis de la LDT "ENTRENAMIENTO". (2020). Objetivos y Estrategias. Quito. 
Equipo de análisis de las LDT "ENTRENAMIENTO". (2020). Matriz de resultados. Quito.

Guaytarilla, C. E. (2020). Modelo de entrenamiento del Ejército de España. Madrid.

Haddock, C., Poston, W., Heinrich, K., Jahnke, S., \& Jitnarin, N. (2016). The Benefits of High-Intensity Functional Training Fitness. Military Medicine, 1508-1514.

Martínez, C. E. (2020). Modelo de entrenamiento del Ejército de Brasil. Brasilia.

Ponce, C. E. (2020). Modelo de entrenamiento del Ejército de EE.UU. Bogotá.

Rodríguez, C. E. (2020). Modelo de entrenamiento del Ejército de EE.UU. Washington. 